\title{
Effectiveness of the Genomics ADvISER decision aid for the selection of secondary findings from genomic sequencing: a randomized clinical trial
}

\author{
Yvonne Bombard, PhD (i) ${ }^{1,2}$, Marc Clausen, $\mathrm{MA}^{2}$, Salma Shickh, MS ${ }^{1,2}$, Chloe Mighton, MSc ${ }^{1,2}$, \\ Selina Casalino, $\mathrm{HBSc}^{2}$, Theresa H. M. Kim, PhD ${ }^{3}$, Sarah M. Muir, BSc ${ }^{2}$, Lindsay Carlsson, $\mathrm{MN}^{4}$, \\ Nancy Baxter, MD PhD ${ }^{1,2,5}$, Adena Scheer, MD MSc, ${ }^{2,6}$, Christine Elser, MD ${ }^{4,6}$, Andrea Eisen, MD, \\ Seema Panchal, $\mathrm{MS}^{8}$, Tracy Graham, $\mathrm{MSc}^{7}$, Melyssa Aronson, $\mathrm{MS}^{8}$, Carolyn Piccinin, $\mathrm{MS}^{8}$, \\ Talia Mancuso, MS ${ }^{7}$, Kara Semotiuk, $\mathrm{MS}^{8}$, Michael Evans, MD², June C. Carroll, MD ${ }^{8,9}$, \\ Kenneth Offit, MD ${ }^{10}$, Mark Robson, MD ${ }^{10}$, Jada G. Hamilton, PhD MPH ${ }^{10}$, Emily Glogowski, $\mathrm{MS}^{11}$, \\ Kasmintan Schrader, MD ${ }^{12,13}$, Raymond H. Kim, MD PhD ${ }^{3,4,6}$, Jordan Lerner-Ellis, PhD ${ }^{8,14}$, \\ Kevin E. Thorpe, MMath ${ }^{15,16}$ and Andreas Laupacis, MD MSc ${ }^{1,2}$, \\ for the Incidental Genomics Study Team
}

\begin{abstract}
Purpose: To evaluate the effectiveness of the Genomics ADvISER (www.genomicsadviser.com) decision aid (DA) for selection of secondary findings (SF), compared with genetic counseling alone.

Methods: A randomized controlled trial (RCT) was conducted to evaluate whether the Genomics ADvISER is superior to genetic counseling when hypothetically selecting SF. Participants were randomized to use the DA followed by discussion with a genetic counselor, or to genetic counseling alone. Surveys were administered at baseline and post-intervention. Primary outcome was decisional conflict. Secondary outcomes were knowledge, preparation for, and satisfaction with decision-making, anxiety, and length of counseling session.
\end{abstract}

Results: Participants $(n=133)$ were predominantly White/European $(74 \%)$, female $(90 \%)$, and $\geq 50$ years old $(60 \%)$. Decisional conflict (mean difference $0.05 ; P=0.60$ ), preparation for decisionmaking $(0.17 ; P=0.95)$, satisfaction with decision $(-2.18 ; P=0.06)$, anxiety $(0.72 ; P=0.56)$, and knowledge of sequencing limitations $(0.14 ; P=0.70)$ did not significantly differ between groups. However, intervention participants had significantly higher knowledge of SF $(0.39 ; \quad P<0.001)$ and sequencing benefits $(0.97 ; P=0.01)$, and significantly shorter counseling time $(24.40$ minutes less; $P<0.001)$

Conclusions: The Genomics ADvISER did not decrease decisional conflict but reduced counseling time and improved knowledge. This decision aid could serve as an educational tool, reducing in-clinic time and potentially health care costs.

Genetics in Medicine (2020) 22:727-735; https://doi.org/10.1038/s41436019-0702-z

Keywords: genomic sequencing; secondary findings; incidental results; decision aid; decision support

\section{INTRODUCTION}

Genomic sequencing (GS) is increasingly used across a range of medical disciplines to improve disease diagnosis and prognosis, as well as to tailor treatment for patients. However, significant barriers exist that hinder the full potential for genomics to advance health care. Aside from the significant challenges in both the analysis and interpretation of results, a major issue is the generation of secondary findings, some of which can be clinically relevant but others may not. ${ }^{1}$ We use the term secondary findings to refer to a broader range of results than those recommended by the American College of Medical Genetics and Genomics (ACMG). ${ }^{2}$ The prevalence of secondary findings is substantial, with cohort studies indicating that the vast majority $(\sim 90 \%)$ of sequenced patients harbor carrier results and pharmacogenetic variants associated with adverse responses to common medications. ${ }^{3}$

Clinical practice guidelines recommend that clinicians should engage in shared decision-making with patients about

\footnotetext{
${ }^{1}$ Institute of Health Policy, Management and Evaluation, University of Toronto, Toronto, ON, Canada; ${ }^{2}$ St. Michael's Hospital, Toronto, ON, Canada; ${ }^{3}$ The Hospital for Sick Children, Toronto, ON, Canada; ${ }^{4}$ University Health Network, Toronto, ON, Canada; ${ }^{5}$ Division of General Surgery, Department of Surgery, University of Toronto, Toronto, ON, Canada; ${ }^{6}$ Faculty of Medicine, University of Toronto, Toronto, ON, Canada; ${ }^{7}$ Sunnybrook Health Sciences Centre, Toronto, ON, Canada; ${ }^{8}$ Mount Sinai Hospital, Sinai Health System, Toronto, ON, Canada; ${ }^{9}$ Department of Family and Community Medicine, University of Toronto, Toronto, ON, Canada; ${ }^{10}$ Memorial Sloan Kettering Cancer Center, New York, NY, USA; ${ }^{11}$ GeneDx, Gaithersburg, MD, USA; ${ }^{12}$ Department of Molecular Oncology and Hereditary Cancer Program, BC Cancer Agency, Vancouver, BC, Canada;

${ }^{13}$ Department of Medical Genetics, University of British Columbia, Vancouver, BC, Canada; ${ }^{14}$ Department of Laboratory Medicine and Pathobiology, University of Toronto, Toronto, ON, Canada; ${ }^{15}$ Dalla Lana School of Public Health, University of Toronto, Toronto, ON, Canada; ${ }^{16}$ Applied Health Research Centre, St. Michael's Hospital, Toronto, ON, Canada. Correspondence: Yvonne Bombard (yvonne.bombard@utoronto.ca). A list of authors and affiliations appears at the end of the paper.
} 
receiving secondary findings before sequencing, particularly for those that are considered medically actionable. ${ }^{4}$ Studies suggest that patients are interested in learning most, if not all, forms of secondary findings. ${ }^{5}$ However, the volume and complexity of secondary findings generated from GS makes engaging in traditional pretest shared decision-making about all secondary findings with each patient unfeasible. Standard genetic counseling, which consists of hours of counseling per test/disease, is highly costly and unfeasible for GS given the thousands of possible secondary findings. ${ }^{6-8}$ As GS use increases, the demand for genetic counselors will exceed their availability. ${ }^{9}$ Novel approaches to patient education and decision support are needed to address the counseling burden, constrained resources, and limited genomics expertise. ${ }^{9}$ There are some existing decision or educational aids on GS for pediatric populations or a narrow range of secondary findings, none of which have been evaluated in a randomized controlled trial (RCT). ${ }^{7,9}$ There are currently no decision aids to guide adult patients' selection of secondary findings from GS that cover the broad range of secondary findings available to patients.

We created an interactive, online decision aid (DA) called the Genomic ADvISER (www.genomicsadviser.com) to guide patients' selection of secondary findings, which has previously been described in detail. ${ }^{10,11}$ Usability testing revealed that participants found the DA acceptable and intuitive to use, with patients indicating that it provided sufficient information for them to reach an informed, values-congruent decision..$^{10}$ Overall, participants expressed positive experiences using the DA, felt that the DA improved their knowledge of GS secondary findings, aided them in decision-making, and was something they would use if actually faced with selecting secondary findings from GS. However, participants also noted that they "would want to talk to a person" before finalizing their decision.

Thus, the Genomics ADvISER offers significant potential to address a critical care gap, especially as GS moves into mainstream medical care. We conducted a superiority RCT to evaluate the effectiveness of the Genomics ADvISER DA compared with genetic counseling alone. We hypothesized that the Genomics ADvISER would reduce patients' decisional conflict (primary outcome) and improve knowledge, satisfaction with decision-making and preparedness for decision-making, and reduce time spent counseling when hypothetically selecting secondary findings.

\section{Design}

\section{MATERIALS AND METHODS}

As described elsewhere, ${ }^{11}$ we conducted a mixed-methods randomized controlled trial (RCT) to evaluate whether our online DA, the Genomics ADvISER, followed by a question and answer (Q\&A) session with a genetic counselor reduced decisional conflict compared with genetic counseling alone when selecting secondary findings. The goal of the study was to evaluate the effectiveness of the DA in pretest counseling. Participants did not undergo genomic sequencing and were not provided with any results; therefore, the study was hypothetical. Pilot or hypothetical trials are recommended by the Ottawa Decision Support Framework (ODSF) Handbook as a means of evaluation of a preliminary version of a DA before a full trial of the final DA in a real-word setting. ${ }^{12}$ Additionally, it has been found that research participants' actual uptake of secondary findings falls within the range of hypothetical uptake reported across studies, ${ }^{13}$ which suggests similarities between hypothetical and real decisions related to learning secondary findings. A subset of intervention participants completed a follow-up qualitative interview to explore their preferences for secondary findings and experience using the DA. Study flow is documented in Supplementary File 1.

The Canadian Institutes of Health Research (CIHR) provided the funding to Y.B. in support of this research (\#143310 \& \#136664). The St. Michael's Hospital (REB \#16052), Mount Sinai Hospital (REB \#16-0054-E), and Sunnybrook Health Science Centre (REB \#198-2016) Research Ethics Boards (REBs) approved this study. Participants provided informed verbal consent. Participants received a $\$ 20$ gift card to a bookstore once they completed their participation in the study. Consolidated Standards of Reporting Trials (CONSORT) guidelines for reporting RCTs were used to guide preparation of this article. ${ }^{14}$

\section{Population}

Participants were recruited from four cancer genetics clinics across three hospitals in Toronto, Canada from September 2016 to March 2018. Eligibility criteria reflected anticipated future clinical eligibility criteria for genomic sequencing. Participants were patients whose personal and/or family histories were suggestive of an inherited cancer syndrome but for whom first-tier genetic testing for a classic causative pathogenic variant (e.g., BRCA1/2) was negative. Genomic sequencing may be used as a second-tier test in identifying the causative variant. ${ }^{15}$ Eligible patients were 18 years of age or older, able to speak and read English, had access to the Internet and to a computer or smart phone. Patients were excluded if they had recurrent metastatic cancer (stage 4), since studies indicate that secondary findings are perceived as burdensome to this population given their ongoing health challenges. ${ }^{16}$ Patients and their family members who participated in the usability study of the DA that preceded this RCT or had previously undergone genomic sequencing were excluded. ${ }^{10}$

Participants from each clinic were randomized using computer-generated randomization in a 1:1 ratio with random permuted blocks of sizes 2 and 4 . Allocations were computer-generated by a biostatistician (K.E.T.) and concealed in envelopes by a team member not involved in the administration of the study, so that the study coordinators administering consents, baseline measures, and randomization were blinded to the participants' allocation. After participants consented and completed baseline measures, the study coordinator opened the randomization envelope to 
reveal the study arm to which the participant was allocated. Given the nature of the study intervention, it was not possible for the study coordinator, principal investigator, or the participant to be blinded to the allocation after randomization.

\section{Intervention arm}

Intervention participants were given a unique login ID and password for the Genomics ADvISER. They used the Genomics ADvISER to select the categories of secondary findings they wished to receive. Secondary findings were "binned" into five categories, based on a previously proposed framework. ${ }^{17}$ Following selection of secondary findings, participants completed all self-administered measures online at time 1 (T1). This was followed by a phone Q\&A session with a genetic counselor who used a standardized script (Supplementary File 2) of questions. At the end of the session, participants completed the same set of online selfadministered measures (T2; Supplementary File 1).

\section{Control arm}

Control participants spoke to a genetic counselor to select their secondary finding categories. Participants were sent a set of standard counseling visual aids developed by the National Society of Genetic Counselors, ${ }^{18}$ which included visuals of cells, genes, chromosomes, and a table summarizing the five categories of secondary findings (Supplementary File 3). This is consistent with standard genetic counseling practice. At the end of the counseling session, participants selected which categories of secondary findings they would like to receive and then completed the online self-administered measures ( $\mathrm{T} 1$; Supplementary File 1).

Six genetic counselors provided counseling for the study. All genetic counselors were board certified and had a minimum of 3 years' experience providing counseling. All counselors received the same training on study conduct and the use of counseling scripts for control and intervention arm participants. All genetic counseling sessions were audio recorded and were periodically checked for fidelity to ensure consistency in the content and delivery of the counseling materials. Counselors kept field notes about each genetic counseling session, detailing any questions participants had, areas that needed further clarification, participants' reasons for their choice of secondary findings, and, in the case of intervention participants, comments about the DA. After 33 participants were enrolled into the study, one counselor (S.S.) provided all genetic counseling for the remainder of the study to facilitate efficient recruitment and data collection.

\section{Outcomes}

The primary outcome was decisional conflict assessed via the validated 16-item Decisional Conflict Scale (DCS; Cronbach's alpha 0.92) ${ }^{19}$ consistent with the Ottawa Decision Support Framework. ${ }^{13}$ The primary time points of comparison were immediately after speaking with a counselor (T1) for the control versus immediately after using the decision aid and speaking with genetic counselor (T2) for the intervention group. Decisional conflict is one of the most common endpoints used in the evaluation of $\mathrm{DAs}^{20}$ since DAs are intended to reduce decisional conflict and increase informed decision-making. ${ }^{19}$ Indeed, promoting informed decisionmaking is a fundamental goal of genetic counseling. As such, decisional conflict is often used as an outcome in the evaluation of decision support tools in genetics and genetic counseling tools specifically. ${ }^{9,21-24}$ The DCS is a reliable and sensitive measure of decisional conflict that can discriminate between decision support interventions. ${ }^{20}$ Each item is scored $1-5$ and a total score on the DCS is calculated by summing all items on the DCS and then dividing by 16 , giving a final score between 1 and $5 .{ }^{25}$ A lower score on the DCS indicates lower level of decisional conflict. The DCS is also converted into a score between 0 and 100, with scores lower than 25 associated with implementing decisions and scores over 37.5 associated with decision delay. ${ }^{13}$

Secondary outcomes included knowledge (administered at baseline [before randomization], T1, and T2), satisfaction with decision-making (T1 and $\mathrm{T} 2$ ), preparation for decisionmaking (T1 and $\mathrm{T} 2$ ), anxiety (baseline, $\mathrm{T} 1$, and $\mathrm{T} 2$ ), and length of counseling session ( $\mathrm{T} 1$ for control and $\mathrm{T} 2$ for intervention). Knowledge was measured using (1) an established 11-item questionnaire consisting of two subscales assessing benefits (Cronbach's alpha 0.70) and limitations (Cronbach's alpha 0.80 ) of genome sequencing, ${ }^{26}$ and (2) a 5item internally developed knowledge questionnaire on secondary findings. Higher knowledge scores on both questionnaires indicate a higher level of knowledge. Satisfaction with decision was measured using the Satisfaction with Decision Scale (Cronbach's alpha 0.86) ${ }^{27}$ and the Preparation for Decision-Making Scale (Cronbach's alpha 0.92$)^{28}$ For both of these scales a higher score signifies a higher level of satisfaction or preparation with a decision. Anxiety was measured using the state subscale of the State-Trait Anxiety Inventory (Cronbach's alpha 0.86 ). ${ }^{29,30}$ Higher scores on the State-Trait Anxiety Inventory indicate higher level of anxiety. Both the control and intervention genetic counseling sessions were recorded to assess the length of the sessions. We also used a single internally developed item to ask intervention participants if they would use the DA to make an actual decision about which secondary findings they would like to learn (Yes, No, Don't Know) at T1 and T2.

\section{Sample size}

Wakefield et al. ${ }^{31}$ reported a standard deviation of 0.6 on the DCS and indicated that a difference of 0.3 was the minimal clinically important difference (MCID). Assuming this, we estimated that 64 patients/arm would be required to have $80 \%$ power at a two-sided significance level of 0.05 to detect the MCID using a standard two-sample $t$-test.

\section{Statistical analysis}

We used descriptive statistics to report participants' baseline characteristics. The analysis of outcomes followed the 
intention-to-treat approach. Mean scores for decisional conflict, knowledge of secondary findings, satisfaction with decision-making, preparation for decision-making, and length of genetic counseling sessions were compared using a $t$-test. We conducted our primary and secondary analyses using the 0-5 scoring system for the DCS based on established methods for analyzing this scale, ${ }^{25}$ and also report $0-100$ scores to indicate which patients fell above and below cut-offs associated with implementing decisions and decision delay. ${ }^{13}$ Knowledge of sequencing benefits and sequencing limitations scores were assessed by summing the number of correct responses to the questions, and compared adjusting for baseline score using analysis of covariance (ANCOVA). Anxiety was assessed by summing participants' responses to the questions and adjusting for baseline score using ANCOVA. Descriptive statistics were used to summarize responses to the question about whether intervention arm participants would use the DA to make an actual decision.

Secondary exploratory analyses examined the impact of the DA alone (T1), without the addition of follow-up counseling, on the study outcomes, and compared intervention and control group scores of decision conflict, knowledge, anxiety, satisfaction, and preparation with decisionmaking at $\mathrm{T} 1$.

Three prespecified subgroup analyses of the primary outcome were performed using linear regression. The subgroups explored were medical history (presence or absence of cancer), baseline knowledge, and baseline anxiety. These subgroups were chosen as we suspected that these factors would have the greatest influence on decisional conflict. Since knowledge and anxiety are both continuous variables and these were exploratory analyses, no assumption of a linear relationship was assumed and restricted cubic splines with three knots were used in the regression models. Since all analyses other than for the primary outcome were considered exploratory and hypothesis generating, no adjustment for multiple comparisons was employed. Statistical analyses were conducted using $\mathrm{R}$, version 3.5.1.

\section{RESULTS}

\section{Participants}

A total of 220 persons were approached for participation, of whom 217 agreed to be contacted by the study team. Of the patients approached, a total of 27 individuals declined to participate, 46 did not respond to contact efforts, and 6 were deemed ineligible after initial contact with the recruiting genetic counselor. One hundred thirty-eight patients consented to participate and were randomized, but 5 patients completed only the baseline session. A total of 133 participants completed all study activities, although 2 intervention participants did not complete the postcounseling survey (Fig. 1). The trial was ended after the target sample size of 128 was achieved, plus an additional 5 cases to account for missing data or drop out.

Participating patients were predominantly female (90\%), well educated (74\%), White/European (74\%), and over 50 years of age $(60 \%)$. Their characteristics are summarized in Table 1.

\section{Outcomes}

The results of the primary and secondary outcomes are shown in Table 2. The Genomics ADvISER did not decrease decisional conflict compared with genetic counseling (mean difference of $0.05,95 \%$ confidence interval [CI] -0.15 to 0.27 ; $P=0.60)$. Median decisional conflict total scores for both arms were below the accepted cut-off score of 25 (intervention: 19.25 [17.16], control: 17.84 [13.51], mean difference -1.41 [ -6.75 to 3.93$] ; P=0.60$ ), which is associated with implementing decisions. ${ }^{32}$ There were no discernible differences in scores for anxiety, knowledge of sequencing limitations, preparation for decision-making, and satisfaction with decisions, though there was a trend toward higher satisfaction with decision for DA users. However, knowledge of secondary findings was significantly higher in the intervention group (mean difference of 0.39 , 95\% CI 0.18 to $0.59 ; P<0.001$ ) as was knowledge of the benefits of GS (mean difference of $0.97,95 \%$ CI 0.22 to $0.95 ; P=0.01$ ). Intervention group participants spent significantly less time on average talking to a genetic counselor (average 16 minutes for the intervention versus 40 minutes for the control (mean difference of 24.40 minutes, $95 \%$ CI -27.72 to -21.07 ; $P<0.001)$.

Secondary analysis comparing $\mathrm{T} 1$ for the control group versus $\mathrm{T} 1$ for the intervention showed no detectable difference in scores for decisional conflict, preparation for decisionmaking, satisfaction with decision-making, anxiety, knowledge of sequencing limitations, and knowledge of secondary findings. However, knowledge of sequencing benefits for the intervention group remained significantly higher than the control group when comparing T1 versus T1 scores (mean difference of $1.0095 \%$ CI of 0.26 to $1.74 ; P=0.01$ ).

Ninety-one percent $(n=60)$ of intervention participants indicated that they would use the DA to make an actual decision about secondary findings. In the subgroup analyses, neither medical history (presence or absence of cancer) nor knowledge (sequencing limitations) exhibited any discernible effect on decisional conflict. On the other hand, anxiety exhibited a nonlinear relationship with decisional conflict $(p=0.031)$ in a linear regression model (i.e., nonlinear association with the log-odds of high decisional conflict) (Fig. 2).

\section{DISCUSSION}

This trial addresses an important gap in clinical genomics and the increasing use of GS across medical specialties: the need for less resource-intense ways of providing genomics education and decision support. As GS becomes part of mainstream clinical care, the number of secondary findings identified will increase, and their management will affect numerous specialties impacted by the diverse range of diseases implicated. Standard genetic counseling is highly costly and unfeasible for GS given the limited number of genetic 


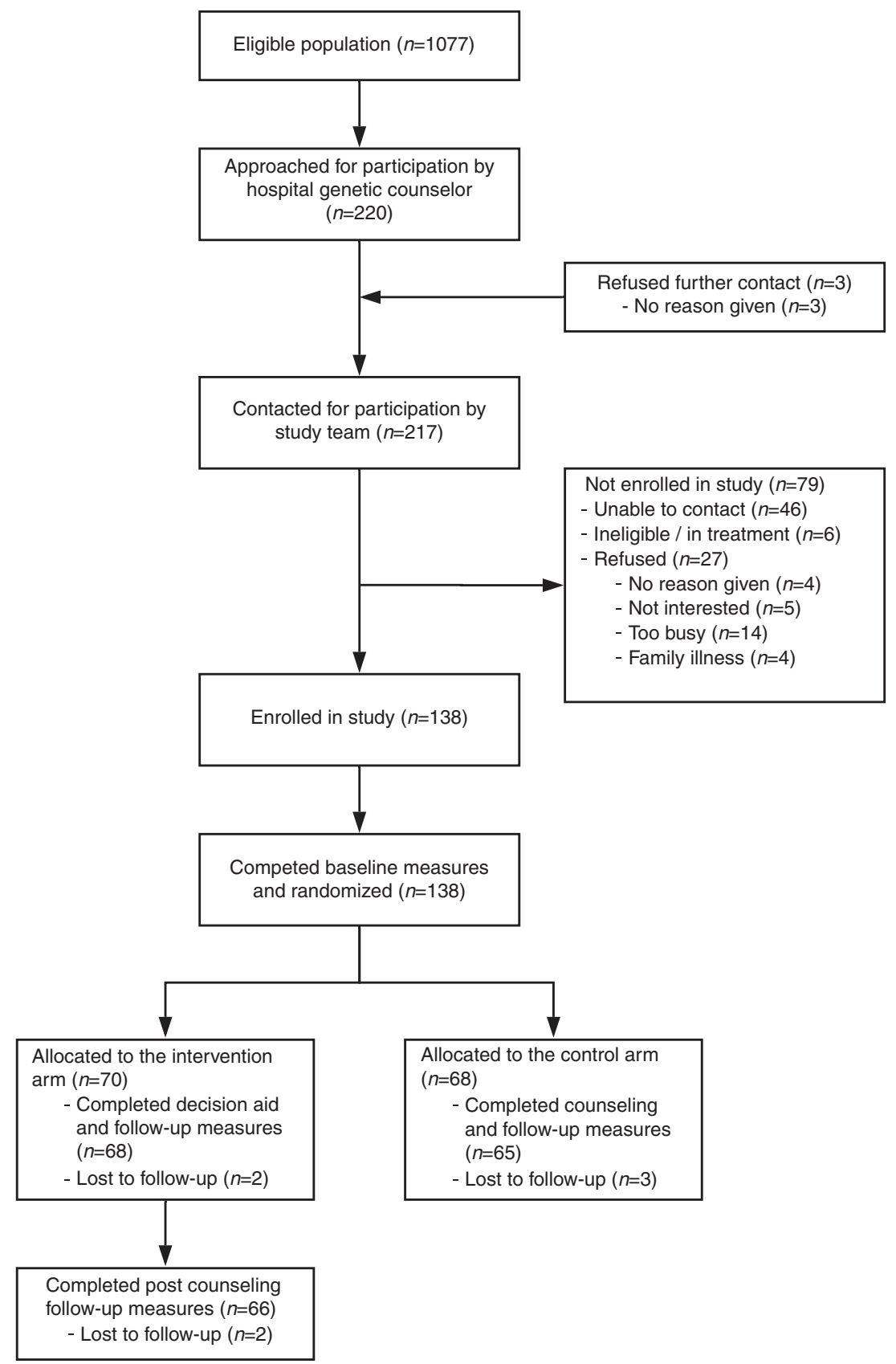

Fig. 1 Consolidated Standards of Reporting Trials (CONSORT) diagram.

counselors and the volume and complexity of secondary findings generated from GS. ${ }^{7,8}$ Our trial results found that the Genomics ADvISER did not decrease decisional conflict compared with genetic counseling alone, but patients who used the decision aid were more knowledgeable and spent less time speaking with the genetic counselor. Participants in both groups reported similar preparation for and satisfaction with their decisions. Thus the Genomics ADvISER is effective and efficient in educating patients about GS and secondary findings, filling a significant gap in light of limited genomics expertise and resources.

Our results are consistent with other studies that have found similar levels of decisional conflict in trials evaluating computerized DAs compared with genetic counseling, ${ }^{24}$ and generally low levels of decisional conflict when deciding about pursuing GS. ${ }^{33}$ There are several reasons that may explain why the Genomics ADvISER was not superior to genetic counseling alone. First, genetic counseling serves two distinct purposes: delivery of genetics education and psychological counseling. ${ }^{21}$ Thus, the impact of a DA on decisional conflict and knowledge are both important attributes. We chose decisional conflict as the primary outcome to calculate sample size because of the availability of estimates for the standard deviation required to detect the MCID in similar trials and populations. However, knowledge is an important outcome as well, especially in this context. The selection of secondary 
findings from GS first requires significant upfront education for patients to make a values-congruent, quality decision. ${ }^{13}$ Indeed, international patient DA evaluation standards and systematic reviews indicate that knowledge is frequently used as the primary outcome in the evaluation of decision aids ${ }^{20}$ and of genetics digital tools specifically. ${ }^{8}$ However, there were no validated genomics knowledge scales at the time of the study, and none that provided sufficient psychometric data. ${ }^{34}$ This significantly hinders comprehensive evaluation of decision aids in genomics, and represents an important area for future research.

Nonetheless, achieving improved genomics knowledge with fewer resources and significantly less time spent with genetic counselors constitutes an important secondary outcome of this study, which is consistent with similar trials ${ }^{35}$ and further bolsters the support for the Genomics ADvISER's effectiveness and efficiency in delivering education and decision support. Comparisons of the impact of the DA alone (without follow-up counseling, T1 versus T1) show that the addition of counseling after the DA did not have an impact on outcomes and did not impact knowledge scores as the benefits of genomic sequencing scores remained significantly higher than the control at the T1 follow up. Therefore, the Genomics ADVISER may prove to be a useful tool for streamlining

Table 1 Participants' sociodemographic and clinical characteristics

\begin{tabular}{|c|c|c|}
\hline Characteristic, number (\%) & $\begin{array}{l}\text { Decision aid } \\
(n=68)\end{array}$ & $\begin{array}{l}\text { Genetic counselor } \\
(n=65)\end{array}$ \\
\hline \multicolumn{3}{|l|}{ Age } \\
\hline$<49$ & $30(44.2)$ & $23(35.4)$ \\
\hline$\geq 50$ & $38(55.8)$ & $42(64.6)$ \\
\hline \multicolumn{3}{|l|}{ Sex } \\
\hline Male & $7(10.3)$ & $6(9.2)$ \\
\hline Female & $61(89.7)$ & $59(90.8)$ \\
\hline \multicolumn{3}{|l|}{ Level of education } \\
\hline$<$ Postgraduate degree & $44(64.7)$ & $41(63.1)$ \\
\hline Postgraduate degree & $24(35.3)$ & $24(36.9)$ \\
\hline \multicolumn{3}{|l|}{ Employment status } \\
\hline Working full time & $31(45.6)$ & $37(56.9)$ \\
\hline$<$ Working part time & $37(54.4)$ & $28(43.1)$ \\
\hline \multicolumn{3}{|l|}{ Income $^{a}$} \\
\hline Prefer not to answer & $10(14.9)$ & $6(9.2)$ \\
\hline$<\$ 80,000$ & $18(26.9)$ & $19(29.1)$ \\
\hline$\$ 80,000+$ & $39(58.2)$ & $39(60.0)$ \\
\hline \multicolumn{3}{|l|}{ Ethnicity } \\
\hline White/European & $54(79.4)$ & $45(69.2)$ \\
\hline Nonwhite/European & $14(20.6)$ & $20(30.8)$ \\
\hline \multicolumn{3}{|l|}{ Affected with cancer ${ }^{a}$} \\
\hline Yes & $44(64.7)$ & $38(59.4)$ \\
\hline No & $23(33.8)$ & $26(40.6)$ \\
\hline
\end{tabular}

aNumbers do not add to 133 because participants had the option to leave the question blank.

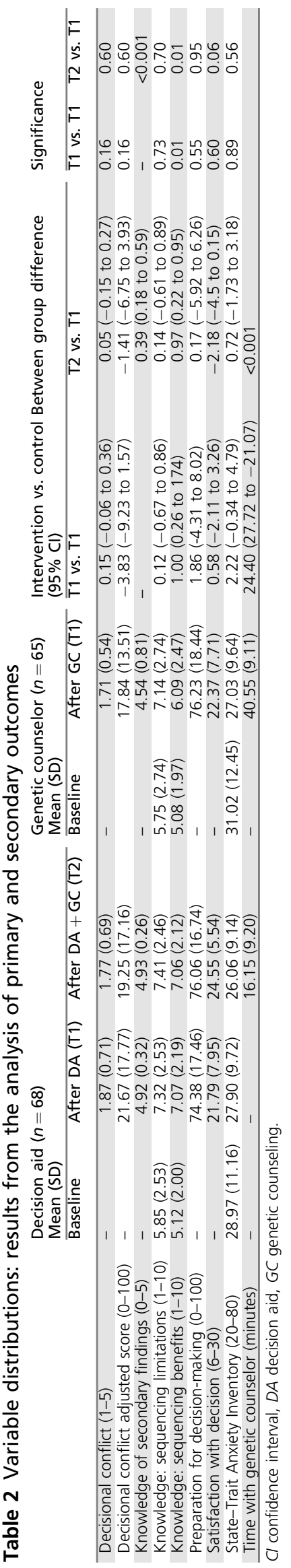




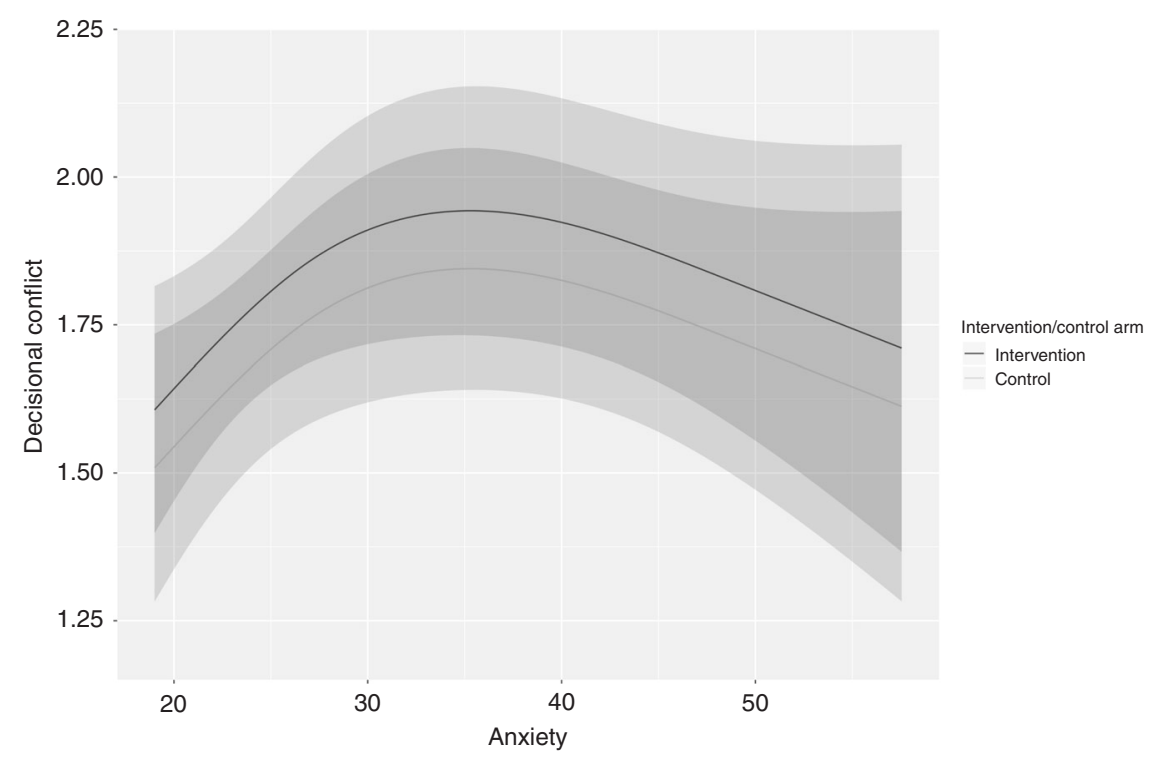

Fig. 2 Subgroup analysis anxiety. Anxiety exhibited a nonlinear relationship with decisional conflict in a linear regression model $(p=0.031)$.

education and counseling, and thus reserving clinical time for shared decision-making.

The reduced time for genetic counseling along with effective educational delivery represent important health service efficiencies, especially in light of the increasing use of GS across medical practices and need for alternate, scalable delivery models of pretest genetic counseling services. In fact, some studies have challenged the requirement for pretest genetic counseling in the context of $\mathrm{GS},{ }^{33,36}$ suggesting the need to identify subsets of individuals who require additional decision support based on their genetic knowledge, health literacy, coping abilities ${ }^{33}$ and cultural factors. ${ }^{37}$ Tailoring support to individuals' needs is critical to identify individuals at risk for making uninformed decisions that fail to align with their core values and preferences, and to avoid disenfranchising others who may benefit from genomic evaluations but feel alienated by a potentially onerous pretest genetic counseling process. ${ }^{33}$ We and others have begun to develop methods identifying patient profiles to tailor pretest genetic counseling $^{38}$ to create further efficiencies, but more work with diverse populations is required to develop and evaluate pragmatic alternatives for pretest genetic counseling services. Tailoring genetic counseling using profiles along with digital decision support tools offer promising strategies to support the large number of patients who will be offered some type of genomic evaluation in the future.

\section{Limitations}

There are several caveats to our trial. This was a hypothetical study; no GS was provided nor were secondary findings returned because the goal of the trial was to evaluate the Genomics ADvISER itself, not the return of secondary findings. Implementing an untested DA among patients making real decisions could result in unknown and potentially harmful outcomes. Additionally, there are currently limited patient populations receiving germline GS and being consistently offered the full spectrum of secondary findings, which makes testing the DA in a population making a hypothetical decision more feasible given the scarcity of patients actually receiving GS. Further, our participants were predominately breast cancer patients who were highly educated and thus our sample consisted of mostly females, and most were over 50 years old. Findings may differ in different populations, such as younger populations, or populations that are predominately male. For instance, males may experience lower decisional conflict than females. ${ }^{39}$ All of our participants had previously undergone genetic testing and counseling for their personal and/or family of cancer and may have possessed higher than average understanding of the limitations and benefits related to genetic testing. However, there were no differences in baseline knowledge scores between the groups. Evaluation of our tool in real-world settings with a larger and more diverse sample is needed. Finally, we only evaluated knowledge immediately after participants completed the DA or genetic counseling session. It is therefore unknown whether participants retained information long term, which may be relevant given that participants actually receiving GS may not receive results until several months after their initial counseling session. Despite these limitations, there are important strengths to our study. We comprehensively assessed outcomes and used an RCT, the gold standard in evaluative design. Thus, our results are highly likely to be internally valid.

\section{Conclusion}

We report results of the effectiveness of a timely patientcentered tool to support clinical delivery of secondary findings from genomic sequencing, filling a significant gap in light of limited genomics expertise and resources. The Genomics ADvISER was not superior to genetic counseling alone, but 
did reduce counseling time and improved knowledge, indicating its effectiveness and efficiency in educating patients about GS and secondary findings, though further research is needed to confirm these findings in more diverse patient populations. Given the shortage of genetics professionals and limited genomics expertise among health-care providers, the Genomics ADvISER can help fill a critical gap in the delivery of GS by serving as an educational tool, reducing in-clinic education time and potentially health-care costs.

\section{SUPPLEMENTARY INFORMATION}

The online version of this article (https://doi.org/10.1038/s41436$019-0702-z)$ contains supplementary material, which is available to authorized users.

\section{ACKNOWLEDGEMENTS}

We thank the following individuals and groups for supporting this study: Nick Watkins, Oana Morar, Jessica Gu, Leslie Ordal, Laura Winter-Paquette, Justin Lorentz, Karen Ott, Yael Silberman, Helios Design Labs, and Reframe Health Films. This study was supported by a Foundation Scheme Grant from the Canadian Institutes of Health Research (\#143310) awarded to Y.B. and the Incidental Genomics Study Team. Y.B. was supported by a New Investigator Award from the Canadian Institutes of Health Research (\#136664). J.G.H., K.O., and M.R. were supported by National Cancer Institute (NCI) P30 CA008748.

\section{DISCLOSURE}

The authors declare no conflicts of interest.

Publisher's note Springer Nature remains neutral with regard to jurisdictional claims in published maps and institutional affiliations.

\section{REFERENCES}

1. Bombard $Y$, Robson $M$, Offit $K$. Revealing the incidentalome when targeting the tumor genome. JAMA. 2013;310:795-796.

2. Kalia SS, Adelman K, Bale SJ, et al. Recommendations for reporting of secondary findings in clinical exome and genome sequencing, 2016 update (ACMG SF v2.0): a policy statement of the American College of Medical Genetics and Genomics. Genet Med. 2017;19:249-255.

3. Parsons DW, Roy A, Yang $Y$, et al. Diagnostic yield of clinical tumor and germline whole-exome sequencing for children with solid tumors. JAMA Oncol. 2016;2:616-624.

4. Green RC, Berg JS, Grody WW, et al. ACMG recommendations for reporting of incidental findings in clinical exome and genome sequencing. Genet Med. 2013;15:565-574.

5. Mackley MP, Fletcher $B$, Parker $M$, Watkins $H$, Ormondroyd $E$. Stakeholder views on secondary findings in whole-genome and wholeexome sequencing: a systematic review of quantitative and qualitative studies. Genet Med. 2017;19:283-293.

6. Tabor HK, Stock J, Brazg T, et al. Informed consent for whole genome sequencing: a qualitative analysis of participant expectations and perceptions of risks, benefits, and harms. Am J Med Genet A. 2012;158A:1310-1319.

7. Birch P, Adam S, Bansback N, et al. DECIDE: a decision support tool to facilitate parents' choices regarding genome-wide sequencing. J Genet Couns. 2016;25:1298-1308.

8. Birch $\mathrm{PH}$. Interactive e-counselling for genetics pretest decisions: where are we now? Clin Genet. 2015;87:209-217.

9. Sanderson SC, Suckiel SA, Zweig M, Bottinger EP, Jabs EW, Richardson LD. Development and preliminary evaluation of an online educational video about whole-genome sequencing for research participants, patients, and the general public. Genet Med. 2016;18:501-512.

10. Bombard $Y$, Clausen $M$, Mighton $C$, et al. The Genomics ADVISER: development and usability testing of a decision aid for the selection of incidental sequencing results. Eur J Hum Genet. 2018; 26:984-995.

11. Shickh S, Clausen M, Mighton C, et al. Evaluation of a decision aid for incidental genomic results, the Genomics ADVISER: protocol for a mixed methods randomised controlled trial. BMJ Open. 2018;8:e021876.

12. O'Connor A. Ottawa Decision Support Framework to address decisional conflict: Ottawa Health Research Institute; 2006.

13. Fiallos $K$, Applegate $C$, Mathews DJH, Bollinger J, Bergner AL, James CA. Choices for return of primary and secondary genomic research results of 790 members of families with Mendelian disease. Eur J Hum Genet. 2017;25:530-537

14. Moher D. CONSORT: an evolving tool to help improve the quality of reports of randomized controlled trials. Consolidated Standards of Reporting Trials. JAMA. 1998;279:1489-1491.

15. O'Daniel JM, Lee K. Whole-genome and whole-exome sequencing in hereditary cancer: impact on genetic testing and counseling. Cancer J. 2012;18:287-292.

16. Miller FA, Hayeems RZ, Bytautas JP, et al. Testing personalized medicine: patient and physician expectations of next-generation genomic sequencing in late-stage cancer care. Eur J Hum Genet. 2014;22:391-395.

17. Berg JS, Adams M, Nassar N, et al. An informatics approach to analyzing the incidentalome. Genet Med. 2013;15:36-44.

18. Johnson K, Kieran S, Riordan S, Dunn B, Nixon K. Genomic counseling guide. Chicago, IL: National Society of Genetic Counselors; 2014.

19. O'Connor AM. Validation of a decisional conflict scale. Med Decis Making. 1995;15:25-30.

20. Stacey D, Légaré $F$, Lewis $K$, et al. Decision aids for people facing health treatment or screening decisions. Cochrane Database Syst Rev. 2014;28: CD001431.

21. Biesecker $B B$, Lewis $K L$, Umstead $K L$, et al. Web platform vs. in-person genetic counselor for return of carrier results from exome sequencing: a randomized clinical trial. JAMA Intern Med. 2018;178:338-346.

22. Green MJ, Peterson SK, Baker MW, et al. Effect of a computer-based decision aid on knowledge, perceptions, and intentions about genetic testing for breast cancer susceptibility: a randomized controlled trial. JAMA. 2004;292:442-452.

23. Kuppermann M, Norton ME, Gates E, et al. Computerized prenatal genetic testing decision-assisting tool: a randomized controlled trial. Obstet Gynecol. 2009;113:53-63.

24. Carlson LM, Harris S, Hardisty EE, et al. Use of a novel computerized decision aid for aneuploidy screening: a randomized controlled trial. Genet Med. 2018;21:923-929.

25. O'Connor AM, Tugwell P, Wells GA, et al. A decision aid for women considering hormone therapy after menopause: decision support framework and evaluation. Patient Educ Couns. 1998;33:267-279.

26. Kaphingst KA, Facio FM, Cheng MR, et al. Effects of informed consent for individual genome sequencing on relevant knowledge. Clin Genet. 2012;82:408-415.

27. Holmes-Rovner M, Kroll J, Schmitt N, et al. Patient satisfaction with health care decisions. Med Decis Making. 1996;16:58-64.

28. Bennett C, Graham ID, Kristjansson E, Kearing SA, Clay KF, O'Connor AM. Validation of a preparation for decision making scale. Patient Educ Couns. 2010;78:130-133.

29. Spielberger C, Gorsuch R, Lushene R, Vagg P, Jacobs G. Manual for the State-Trait Anxiety Inventory. Palo Alto, CA: Consulting Psychologists Press; 1983.

30. Julian LJ. Measures of anxiety: State-Trait Anxiety Inventory (STAI), Beck Anxiety Inventory (BAI), and Hospital Anxiety and Depression ScaleAnxiety (HADS-A). Arthritis Care Res (Hoboken). 2011;63(Suppl 11): S467-S472.

31. Wakefield CE, Meiser B, Homewood J, et al. A randomized controlled trial of a decision aid for women considering genetic testing for breast and ovarian cancer risk. Breast Cancer Res Treat. 2008;107:289-301.

32. O'Connor A. User manual: decisional conflict scale. Ottawa Hospital Research Institute; 1993 https://decisionaid.ohri.ca/docs/develop/ User_Manuals/UM_Decisional_Conflict.pdf [Updated 2010].

33. Pacyna JE, Radecki Breitkopf $\bar{C}$, Jenkins SM, et al. Should pretest genetic counselling be required for patients pursuing genomic sequencing? Results from a survey of participants in a large genomic implementation study. J Med Genet. 2018;56:317-324. 
34. Sanderson SC, Loe BS, Freeman M, et al. Development of the Knowledge of Genome Sequencing (KOGS) questionnaire. Patient Educ Couns. 2018;101:1966-1972.

35. Wang C, Gonzalez R, Milliron KJ, Strecher VJ, Merajver SD. Genetic counseling for BRCA1/2: a randomized controlled trial of two strategies to facilitate the education and counseling process. Am J Med Genet A. 2005;134a:66-73.

36. Sutton EJ, Kullo IJ, Sharp RR. Making pretest genomic counseling optional: lessons from the RAVE study. Genet Med. 2018;20:1157-1158.
37. Vadaparampil ST, Cragun D. Shared decision making: Implications for return of results from whole-exome and whole-genome sequencing. Transl Behav Med. 2018;8:80-84.

38. Mighton C, Carlsson L, Clausen M, et al. Development of patient "profiles" to tailor counseling for incidental genomic sequencing results. Eur J Hum Genet. 2019;27:1008-1017.

39. Graham ME, Westerberg BD, Lea J, et al. Shared decision making and decisional conflict in the management of vestibular schwannoma: a prospective cohort study. J Otolaryngol Head Neck Surg. 2018;47:52.

\section{for the Incidental Genomics Study Team}

Yvonne Bombard, PhD (PI) ${ }^{1,2}$, Susan Armel, MSc MS MScCH(Ed) ${ }^{4,17}$, Melyssa Aronson, $\mathrm{MS}^{8,17}$, Nancy Baxter, MD PhD ${ }^{1,2,5}$, Ahmed Bayoumi ${ }^{1,2}$, Ken Bond ${ }^{18}$, June C. Carroll, MD ${ }^{8,9}$, Timothy Caulfield, LLB LLM ${ }^{19,20,21}$, Marc Clausen, MA ${ }^{1,2}$, Tammy Clifford, PhD ${ }^{22}$, Iris Cohn, MSC RPh ${ }^{3}$, Irfan Dhalla, MD MSc ${ }^{1,2,23,24,25}$, Craig C. Earle, MD MSc ${ }^{25}$, Andrea Eisen, MD ${ }^{7}$, Christine Elser, MD ${ }^{4,8,25}$, Michael Evans, MD2 , Tracy Graham, MSc ${ }^{7}$, Emily Glogowski, MS ${ }^{11}$, Jada G. Hamilton, PhD MPH ${ }^{10}$, Wanrudee Isaranuwatchai, $\mathrm{PhD}^{1,2}$, Monika Kastner, PhD ${ }^{1,2,9}$, Raymond H. Kim, MD PhD ${ }^{3,4,25}$, Andreas Laupacis, MD MSc ${ }^{1,2}$, Jordan Lerner-Ellis, $\mathrm{PhD}^{8,14}$, Michelle Mujoomdar, $\mathrm{PhD}^{26}$, Kenneth Offit, $\mathrm{MD}^{10}$, Seema Panchal, $\mathrm{MS}^{8}$, Mark E. Robson, $\mathrm{MD}^{10}$, Adena Scheer, MD MSc ${ }^{2,5}$, Stephen W. Scherer, $\mathrm{PhD}^{3,17,25}$, Kasmintan Schrader, $\mathrm{MD}^{13,27}$, Terrence Sullivan, $\mathrm{PhD}^{1}$ and Kevin E. Thorpe, MMath ${ }^{15,28}$

${ }^{17}$ Department of Molecular Genetics, University of Toronto, Toronto, ON, Canada; ${ }^{18}$ Institute of Health Economics, Edmonton, AB, Canada; ${ }^{19}$ Faculty of Law, University of Alberta, Edmonton, AB, Canada; ${ }^{20}$ School of Public Health, University of Alberta, Edmonton, AB, Canada; ${ }^{21}$ Health Law Institute, University of Alberta, Edmonton, AB, Canada; ${ }^{22}$ School of Epidemiology and Public Health, University of Ottawa, Ottawa, ON, Canada; ${ }^{23}$ Health Quality Ontario, Toronto, ON, Canada; ${ }^{24}$ Institute for Clinical Evaluative Sciences, Toronto, ON, Canada; ${ }^{25}$ Department of Medicine, University of Toronto, Toronto, ON, Canada; ${ }^{26} \mathrm{Canadian}$ Agency for Drugs and Technologies in Health, Ottawa, ON, Canada; ${ }^{27} \mathrm{BC}$ Cancer Agency, Vancouver, BC, Canada; ${ }^{28} \mathrm{Applied} \mathrm{Health}$ Research Centre (AHRC), Li Ka Shing Knowledge Institute of St. Michael's Hospital, Toronto, ON, Canada 